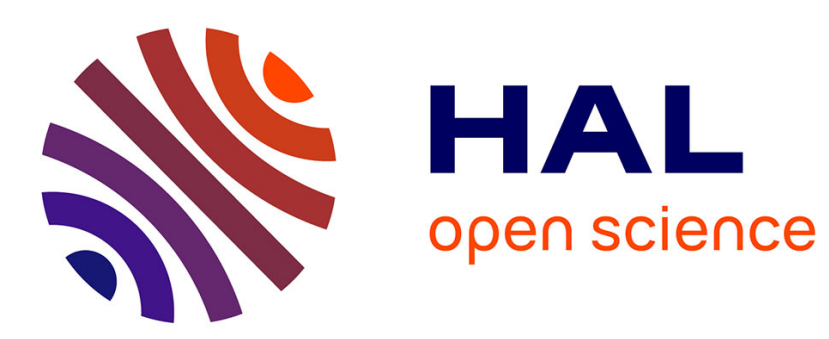

\title{
Les historiens et le nazisme. Pratiques historiographiques, légitimation et engagement
}

Christian Ingrao

\section{To cite this version:}

Christian Ingrao. Les historiens et le nazisme. Pratiques historiographiques, légitimation et engagement. Sociétés contemporaines, 2000, 39 (1), pp.79-83. 10.3406/socco.2000.1802 . halshs-03101207

\section{HAL Id: halshs-03101207 \\ https://shs.hal.science/halshs-03101207}

Submitted on 7 Jan 2021

HAL is a multi-disciplinary open access archive for the deposit and dissemination of scientific research documents, whether they are published or not. The documents may come from teaching and research institutions in France or abroad, or from public or private research centers.
L'archive ouverte pluridisciplinaire HAL, est destinée au dépôt et à la diffusion de documents scientifiques de niveau recherche, publiés ou non, émanant des établissements d'enseignement et de recherche français ou étrangers, des laboratoires publics ou privés. 


\section{Les historiens et le nazisme. Pratiques historiographiques, légitimation et engagement.}

Que peut-il y avoir de commun entre Theodor Schieder et Hans-Joachim Beyer? Le premier, historien très connu en Allemagne (mais non traduit en France), a formé de grands ténors de l'histoire sociale actuelle, tel Hans Ulrich Wehler ou Wolfgang Mommsen. Le second n'a pas eu la brillante carrière scientifique du premier. Ils sont pourtant tous les deux des représentants de l'historiographie allemande telle qu'elle se pratique entre les deux guerres à Königsberg, sous la direction de Hans Rothfels. Il n'en reste pas moins que Hans-Joachim Beyer, après la soutenance d'une thèse d'histoire, intègre le $\mathrm{SD}^{1}$, où il prend en charge un travail d'expertise au service des politiques raciales en instance de mise en oeuvre, alors que Schieder se tourne vers la carrière universitaire avec le succès que l'on sait. Les deux itinéraires, commencés sous les mêmes auspices, n'ont bien entendu pas la même signification. Il n'en reste pas moins qu'une frappante convergence dans les lieux d'études, les centres d'intérêts scientifiques, ainsi qu'un certain engagement de la pratique historique dans le champs politique amène à poser le problème des liens entre historiens et nazisme, entre appareil d'état et réseaux scientifiques, entre paradigmes historiographiques de l'Entre-deux-guerres et construction idéologique. C'est cette problématique qui forme le noyau des travaux de Peter Schöttler.

Tout à la fois spécialiste des historiographies française et allemande des années 30 et protagoniste des principaux débats méthodologiques actuels, Peter Schöttler s'est penché sur cette question à l'occasion d'une conférence au Congré des Historiens de Francfort, ainsi que dans un ouvrage collectif consacré à l'engagement de l'historiographie allemande dans la politique révisionniste de la République de

\footnotetext{
${ }^{1}$ Sicherheitsdienst, service de sécurité des SS. Il s'agit de la centrale d'espionnage intérieur et extérieur du Troisième Reich.
} 
Weimar et du Troisième Reich des années 20 et $30 .^{2}$ Cherchant à évaluer le rapport au politique de la pratique historienne, il a ainsi pu esquisser trois axes de recherches. En premier lieu, un certain nombre de travaux ont souligné le rôle de réseaux scientifiques dans la mise en place de liens entre historiens et appareil étatique. En second lieu, en développant lors du Congrés de Francfort un questionnaire très novateur - mais pas isolé- portant sur une re-définition du nazisme, il a pu souligner la multiplicité des situations et des rapports particuliers des historiens au nazisme, tel qu'il s'incarne dans ses discours et ses pratiques. Enfin, au travers de l'exemple de Franz Petri, il a pu montrer comment les travaux particuliers d'un historien ont pu inspirer telle ou telle partie de la politique nazie.

\section{Des historiens dans le Nazisme? Les réseaux de l'histoire engagée.}

De fait, l'un des grands mérites de l'ouvrage collectif dirigé par Peter Schöttler est d'étudier des instiutions de recherche dont l'existence avait été plus ou moins refoulée par la communauté des historiens allemands jusque dans les années 90. Qui, en effet, voulait ou pouvait se souvenir du gigantesque projet d' "Encyclopédie de la germanité des frontières et de l'étranger" ou même de l'existence des Volksdeutsche Forschungsgemeinschaften, ces groupes de recherche au statut assez opaques, puisque de droit privé tout en étant financées à couvert par les ministères de l'Intérieur et des Affaires étrangères?

Il s'agit pourtant bien là des réseaux les plus important de l'engagement des historiens de l'Entre-deux-guerres dans ce qu'ils percevaient sans doute alors comme une rénovation historiographique et, au-delà même de l'enjeu paradigmatique, comme une forme d'engagement qui était de l'ordre du sentiment national.

Les Volksdeutsche Forschungsgemeinschaften ont assurément été créées dans le contexte de la République de Weimar et ne peuvent être considérées comme étant d'essence proprement nazie. Il n'en reste pourtant pas moins qu'elles ont continuellement contribué à soutenir une politique de révision du traité de Versailles en fondant scientifiquement les prétentions allemandes à la restitution des territoires perdus lors de la Paix de Versailles. Alors qu'Hans Rothfels et ses élèves démontrent à l'Université de Königsberg la germanisation précoce des territoires de la Baltique, les colloques annuels de la Westdeutsche Forschungsgemeinschaft abordent en 1931 "la défense de l'espace occidental de l'aire culturelle germanique", tandis qu'ils se penchent en 1935 sur la question sensible des "Allemands en France, [et des]Français en Allemagne", ainsi que sur la "mission culturelle [allemande] dans l'Allemagne du

\footnotetext{
2 Peter Schöttler, "Von der rheinischen Landesgeschichte zur nazistischen Volksgeschichte- oder die "unhörbare Stimme des Blutes.", communication au Congrés des historiens allemands, Francfort, 10 septembre 1998 ; Peter Schöttler (éd.), Geschichtsschreibung als Legitimationswissencschaft 1918-1945, Francfort, Suhrkamp, 1998, 344 pages.
} 
nord-ouest et les Pays-Bas". Autant de thèmes propices à la légitimation d'une restitution des territoires d'Eupen et de Malmédy perdus à Versailles et, plus généralement, d'une présence allemande forte sur ses frontières occidentales.

Cette Westdeutsche Forschungsgemeinschaft connait à partir de 1934 tout comme les autres Forschungsgemeinschaften une nazification rapide, culminant pendant la guerre avec la prise en main des Volkstumswissenschaften par le Reichssicherheitshauptamt (RSHA), organisme qui centralise les organes de répression SS, SD et Gestapo en particulier. ${ }^{3}$

$\mathrm{Au}$ total, les contributions de l'ouvrage collectif donne une impression de foisonnement des structures qui semblent avoir eu une activité éditoriale importante. Des organisations dynamiques, qui, si elles sont diverses, se reconnaissent toutes dans une écriture de l'histoire engagée au service de la révision des traités et, plus généralement, au service d'une Allemagne qu'un consensus latent décrit comme amoindrie. Si ces réseaux ne peuvent être considérés comme nazis dans les années 20 et le début des années 30, les points communs entre une historiographie prenant le peuple (Volk) pour acteur de l'histoire et le fondamentalisme nazi basé sur le déterminisme racial étaient trop nombreux pour que la nazification posât des problèmes particuliers. Si jusqu'ici l'apport de la communauté historienne à l'activité du Troisième Reich avait été si largement sous-estimé, c'est bien, sur le plan scientifique, parce que les réseaux de sociétés de Volksforschung étaient restés inexplorés : ils sont la véritable courroie de transmission entre les membres d'un corps universitaire que l'on a longtemps voulu croire épargné par la marée brune et une hiérarchie nazie que l'on s'est trop longtemps représentée comme un quarteron de brutes épaisses présidant par la magie de la rhétorique aux destinées d'une nation de 80 millions de personnes.

\section{Les historiens et le nazisme : l'attractivité d'un système de croyances.}

Dans sa conférence de Francfort, Peter Schöttler entend d'autre part poser à plat la question du rapport au nazisme des historiens. Que signifie la "participation" d'historiens au nazisme : "simplement" un travail de formulation idéologique, de propagande ou bien une action concrête? Plus profondément peut-être, quels sont les critères permettant de dire que tel article ou tel ouvrage est d'inspiration nazie? Qu'est-ce, enfin, que le nazisme : une idée politique, une idéologie, un discours, une

\footnotetext{
3 Peter Schöttler, "Die historische 'Westforschung' zwischen 'Abawehrkampf' und territorialer Offensive.”, in Geschichtsschreibung, Op. Cit., p. 204-262, ici p.209. Sur le RSHA, on se reportera à la synthèse de Norbert Frei, L'Etat Hitlerien et la société allemande, Seuil, Paris, 1994, 400 pages, particulièrement pp. 167-179 ou à Pierre Ayçoberry, La société allemande sous le Troisième Reich, Seuil, Paris, 1998, 435 pages, pp. 38-42 et en ce qui concerne son rôle dans la mise en place du génocide, Michael Wildt, “Avant la 'Solution Finale'. La politique juive du Service de la Sécurité de la SS, 1935-1938.”, in Genèse, n²4, Paris, 1995, 23 pages.
} 
mentalité? Ces questions, pour abstraites qu'elles paraissent, permettent de dresser un état nuancé de la question tenant compte de la diversité des situations, de la complexité des comportements.

Le cas des élèves de Hans Rothfels permet de bien comprendre l'enjeu du rapport au nazisme des historiens. Tous ont adhéré au projet d'historiographie völkisch développé par le professeur de Königsberg, mais le rapport au nazisme s'est développé de manière différentielle et complexe pour chacun. Si Werner Conze a connu une carrière assez classique d'historien conservateur, Karl-Heinz Pfeffer s'est pleinement engagé dans une activité de légitimation de l'expansionnisme nazi, tandis que d'autres élèves de Rothfels à Königsberg, certes bien moins liés à lui, ont intégré la SS et le RSHA et ont fourni un travail de formulation dogmatique légitimant les politiques génocides. Le cas, étudié par Kar-Heinz Roth, de Hans Joachim Beyer, qui fit une partie de ses études à Königsberg du temps de Rothfels et servit de conseiller à l'un des Einsatzgruppen illustre très bien cet engagement total au service du fondamentalisme génocidaire. ${ }^{4}$ Toute tentative de tableau d'ensemble du rapport au nazisme de la commuauté historienne pourrait achopper sur cette diversité des situations. Peter Schöttler propose - à l'occasion de l'étude du cas de Franz Petri, l'un des dirigeants de la Westdeutsche Forchungsgemeinschaft - de la dépasser en étudiant les invariants de la pratique historique de la Volksforschung des années 20 et 30. Pour lui, en effet, les historiens de cette époque "se meuvent dans un univers mental dans lequel le "Peuple", la "Race", le "Sang", le Sol" constituent des concepts constitutifs formant une horizon de pensée à tout moment compatible avec le nazisme. Les symboles collectifs langagiers [qui se trouvent dans les écrits de Petri] forment un a-priori discursif incapable de susciter une quelconque résistance à l'idéologie raciste et antisémite nazie." Peter Schöttler pose en d'autres termes le problème de l'existence de soubassements mentaux transcendant la "simple" adhésion consciente et matérielle au racisme nazi. Point n'est donc besoin d'être adhérent au NSDAP pour partager l'imaginaire nazi.

Se situant au-delà d'une trop classique histoire des idées qui n'a pas grand chose à apporter à l'histoire du nazisme, Peter Schöttler esquisse au travers de l'exemple des historiens un modèle d'étude des processus d'appropriation de certaines dimensions du système de croyances nazi par des groupes sociaux. Les historiens, en l'occurence, partageraient avec les cohortes brunes la conviction d'une menace par ceux que l'on appelle les "allogènes" des minorités allemandes laissées hors des frontières du Reich par les traités de 1919, menace qui ne peut être conjurée que par une politique de révision des traités : le nazisme, examiné par ce prisme, ne peut-il se voir conféré par les Akademiker des Historische Seminaren à tout le moins

\footnotetext{
${ }^{4}$ Karl-Heinz Roth, "Heydrichs Professor : Historiographie des 'Volkstums' und der Massenvernichtungen. Der Fall Hans Joachim Beyer.", in Peter Schöttler (éd.), Geschichtsschreibung als Legitimationswissenschaft, 1918-1945, pp. $262-342$
} 
l'attrait d'une intransigeance "révisionniste" salvatrice? Les parcours des élèves de Rothfels, de Schieder à Hans-Joachim Beyer, ne feraient ainsi que refléter les différentes réactions à cette attractivité, de l'expression d'une communauté de préoccupations dans le cadre d'une pratique historiographique légitimant des aspirations politiques pour l'un, à un engagement sans concessions dans les pratiques génocidaires pour le second.

\section{L'histoire et le nazisme : volonté nazie et ambition historienne d'une légitimation historiographique.}

Le dernier des axes de recherche développés par Peter Schöttler tient au statut même de l'histoire et, partant, de l'historien au sein du Troisième Reich. La production des Volksdeutsche Forschungsgemeinschaften a connu une publicisation poussée dans l'Allemagne des années 30 et 40 . Les thèses, ouvrages et enquêtes collectives trouvent financements et maisons d'éditions. Plus profondément, l'histoire connait un regain d'intérêt, enseignée qu'elle est jusque dans les cours prodigués aux futurs fonctionnaires du RSHA. C'est bien, selon Peter Schöttler, parce que l'histoire a acquis sous le Troisième Reich une dimension légitimatrice non négligeable.

L'exemple des travaux de Franz Petri fournit ici un exemple saisissant de cette dimension légitimatrice mais aussi de l'impact qu'a pu connaitre la pratique historique dans les cercles de pouvoir nazis.

Franz Petri publie ainsi en 1935 sa thèse d'habilitation sur l' "héritage national germanique en Wallonie et dans le Nord de la France". L'ambition de l'ouvrage est de donner de nouveaux fondements à l'histoire du peuplement et des mouvements de populations de l'Europe du Nord-Ouest. Franz Petri s'attache ainsi à démonter la cohérence entre données archéologiques et onomastiques d'une part et carte raciologique d'autre part. Il donne, nollens vollens, en ce domaine une base historiographique au déterminisme racial nazi, illustrant ainsi parfaitement la fonction légitimatrice acquise par l'histoire dans le nouveau régime.

Lorsqu'en Juin 1940, le SS-Brigadeführer et secrétaire d'État au ministère de l'Intérieur Wilhelm Stuckart est chargé de produire un projet de frontière du Reich avec la France vaincue, il se réfère nommément à l'ouvrage de Petri pour donner un fondement historique à un tracé incorporant au Reich les territoires situés au nord d'une ligne courant de la Somme au Jura. En 1942, Hitler lui-même justifie sa politique d'incorporation de territoires occidentaux en citant les travaux de Franz Petri.

Sans faire de l'ouvrage de Petri un idéal-type du livre d'inspiration nazi, Peter Schöttler souligne ainsi les référents symboliques et imaginaires communs à la Volksgeschichte et au système de croyances nazi, mais aussi la propention nazie à chercher dans l'histoire les indices du déterminisme racial. L'histoire devient alors le 
champs scientifique dans lequel s'incarne et se prouve l'immanence des fondements biologiques de la vision du monde nazie.

Au total, les pistes de recherches ouvertes par Peter Schöttler et les auteurs du recueil d'articles ouvrent, au-delà des enjeux mémoriels propres à la communauté historienne et des enjeux civiques propres à l'Allemagne des années $90^{5}$, des perspectives prometteuses pour l'histoire des historiens et de la pratique historiographique, mais aussi pour celle du nazisme. L'étude de la position spécifique des réseaux d'historiens allemands, située à l'interface entre production du savoir et pratiques étatiques génocidaires et l'examen des modes d'intériorisation de l'idéologie national-socialiste d'un groupe d'intellectuels permettront, à n'en pas douter, de jeter une lumière crue sur ce qui a été un imaginaire nazi et qui aurait pu devenir une mémoire nazie.

\author{
Christian Ingrao \\ Centre Marc Bloch (Berlin)
}

\footnotetext{
${ }^{5}$ Ces enjeux ne sont pas à négliger, en témoigne les réactions épidermiques des discutants au Congré des Historiens ainsi que le plaidoyer en faveur de Schieder prononcé lors d'une conférence à Berlin et reproduit dans la Frankfurter Allgemeine Zeitung du Lundi 4 janvier 1999, p.48 par Hans Ulrich Wehler, et le commentaire de cette conférence par Gustav Seibt dans la Berliner Zeitung des 12 et 13 décembre 1998, p.9. Il n'en reste pas moins que ces débats ne doivent pas masquer les enjeux scientifiques des travaux abordant le rôle de l'histoire et des historiens sous le Troisième Reich.
} 